\title{
Voix et Images
}

\section{Notes}

\section{Maximilien Laroche et Jacques Allard}

Volume 12, numéro 1 (34), automne 1986

Québec-Amérique latine

URI : https://id.erudit.org/iderudit/200621ar

DOI : https://doi.org/10.7202/200621ar

Aller au sommaire du numéro

Éditeur(s)

Université du Québec à Montréal

ISSN

0318-9201 (imprimé)

1705-933X (numérique)

Découvrir la revue

Citer ce document

Laroche, M. \& Allard, J. (1986). Notes. Voix et Images, 12(1), 166-170.

https://doi.org/10.7202/200621ar

Ce document est protégé par la loi sur le droit d'auteur. L'utilisation des services d'Érudit (y compris la reproduction) est assujettie à sa politique d'utilisation que vous pouvez consulter en ligne.

https://apropos.erudit.org/fr/usagers/politique-dutilisation/
Cet article est diffusé et préservé par Érudit.

Érudit est un consortium interuniversitaire sans but lucratif composé de l’Université de Montréal, l'Université Laval et l'Université du Québec à Montréal. Il a pour mission la promotion et la valorisation de la recherche. https://www.erudit.org/fr/ 


\title{
Notes
}

\author{
par Maximilien Laroche et Jacques Allard
}

\section{Cadernos de CEF}

no 1, Décembre 1984/no 2, Mars 1985, Universidade Federal Fluminense, Niterà, Brésil.

Le "circulo de estudos francofonos» (CEF) de l'Universidade Federal Fluminense, à Niterà, au Brésil, à peine fondé, en mars 1984, a lancé, presque coup sur coup, les deux premiers numéros de sa revue, Os cadernos do CEF.

Le numéro 1 (décembre 1984) "Amérique latine et Afrique" présente un sommaire copieux. On y relève des études comparatives sur les littératures latino-américaines: "Similitudes et différences entre l'Amérique hispanophone et l'Amérique lusophone" par Lilian Pestre de Almeida; "les Figures du Patriarche et du marron dans 3 romans de la Caraibe et de l'Amérique latine" par Maximilien Laroche; "Dona Flore seus dois maridos de Jorge Amado et le Mat de cocagne de René Depestre" par Jayme Kopke; «Une analyse d'un poème de Césaire" par Maria Bernadette Velloso Porto; "Des traductions en portugais de poèmes d'Afrique francophone" par Lilian Pestre de Almeida et Marilia Cruz Claramunt. Et comme document: un texte d'Eliane Zagury sur «l'Espagnol littéraire de l'Amérique: l'exemple de Porto-Ricon. Notons aussi dans la section "compte-rendu", une recension de l'Anthologie de la nouvelle poésie créole de Lambert-Félix Prudent, que fait Euridice Figueiredo et qui relève avec beaucoup de pertinence les contradictions et les handicaps dont cet ouvrage porte un témoignage de manière directe ou indirecte.

Le numéro 2 (mars 1985) «Le Québec vu du Brésil» fait la part belle à l'œuvre de Jacques Ferron. En effet, trois études lui sont consacrées: «Temps, masque et identité dans Lorenzaccio de Musset et la Tête du roi de Jacques Ferron " par Jayme Kopke; "la Laine et le crin", un conte de Jacques Ferron, tissage de la vie et de la mort par Gloria Miranda da Silva; "le Petit chaperon rouge de Perrault à Ferron: un trajet intertextuel» par Jayme Kopke. À cela s'ajoute dans la section des études comparatives: "la Via crucis du corps: lecture intertextuelle d'Anne Hébert et de Clarice Lispector" par Maria Bernadette Velloso Porto; un texte d'Euridice Figueiredo: «Pour une approche contrastive en civilisation ou la question de la langue au Québec et au Brésil»; et une analyse de Lilian Pestre de Almeida: «l'Image de la France et des Français au miroir du Brésil". La section des traductions nous offre, cette fois-ci, des traductions en portugais de poèmes de Gaston Miron par Flavio Aguiar et d'Anne Hébert par Lilian Pestre de Almeida. Dans la section «compterendu ", Euridice Figueiredo fait la revue critique de Visions du Québec, texte et image.

La nouveauté dans ce deuxième numéro est dans la publication d'un long entretien de l'équipe de rédaction de la revue avec Noël Audet, poète, 
romancier et professeur à l'UQAM, qui, dans le cadre des échanges entre le Québec et le Brésil, avait effectué une session d'enseignement à l'Universidade Federal Fluminense.

Cette variété de textes publiés par des universitaires brésiliens ayant une longue pratique de l'enseignement et de la recherche dans le domaine des littératures francophones, l'approche comparatiste de ces textes, et la volonté d'allier à une approche critique distanciée la saisie "ao vivo» de la parole même des créateurs ou critiques francophones par des entrevues, suffiraient déjà à faire reconnaître le mérite de ces Cadernos do CEF. Mais nous devons signaler et encourager l'ouverture de la politique éditoriale que semble décidée à mener l'équipe de rédaction de la revue, animée par Lilian Pestre de Almeida également correspondante de Voix et images au Brésil. En plus de se doter d'un comité de lecture dont les membres ont été choisis sans aucun doute afin de représenter un large éventail de chercheurs du Québec et de l'Amérique latine, dans le domaine des études francophones, la revue se propose un programme de publications sur des thèmes très suggestifs: «Poésie caribéenne; oralité et création; écrire au féminin ». Elle se propose de plus de faire une large place à la collaboration extérieure. En publiant dans Chemins actuels, la revue des professeurs mexicains de français, «la Carte d'Ulysse ou l'exercice de lecture d'un conte de Jacques Ferron", Lilian Pestre a particulièrement insisté sur la nécessité d'une collaboration des professeurs hispanoaméricains aux Cadernos do CEF.

La revue, par l'intérêt porté à la coopération culturelle avec le Québec, et par la participation contrastée de chercheurs de toute l'Amérique entend donc mettre l'accent sur l'étude de la francophonie américaine.

Mis à part des problèmes de typographie (caractères beaucoup trop petits, impression trop pâle) qui rendent difficile la lecture des textes, il n'y a que des éloges à faire aux Cadernos do $C E F$, pour ces deux premiers numéros.

M.L.

\section{Canadian literature}

Université de la Colombie britannique, 2029 West Mall, Vancouver, V6T IW5 no 108, printemps 1986 (Popular Culture)

Sous-titrée «Littérature canadienne» (voir en page 1), la grande revue de «UBC» continue d'afficher sa curiosité québécoise. Dans ce fort numéro consacré à la culture populaire au Canada (du phénomène Harlequin aux histoires terre-neuviennes, en passant par P. Berton et les magazines), on fait une place particulière à nos feuilletons télé: «Les téléromans: un phénomène québécois" (par Solange Chaput-Rolland); et à la chanson: "Chanter est un pays" (par Bruno Roy). On trouve ensuite plusieurs recensions d'ouvrages: le Phénomène IXE-13 (Pr. de Laval), les Aires de la chanson québécoise (Ed. Triptyque), Selected Tales of Jacques Ferron (Anansi), Haiku: Anthologie canadienne (Asticou), et Lettres d'une autre (L'Hexagone). Enfin, une notice nécrologique consacrée à Michel Beaulieu (1941-1985) par R. Giroux. 


\section{Francofonia}

Université de Bologne, Dipartimento di L.L.S.M. - Filologia Romanza, via Zamboni 38, 40126 Bologna Italia

no 9, automne 1985

La remarquable revue de Liano Petroni a maintenant cinq ans. C'est dans cette revue italienne (et non française ou québécoise ou belge) que les littératures d'expression française sont étudiées ensemble, comme si cela allait de soi! Dans la première section ("Saggi e Studi») après des entretiens avec Nathalie Sarraute, viennent en italien: une étude de Liana Nissim consacrée à un poème de Gilles Hénault, «Temps des aurores du temps »; en français: "les Leurres littéraires chez Pierre Gérin " par Grazia Merler. Puis un essai de Giorgio De Piaggi sur l'écriture féminine en francophonie (bien informé sur le Québec). On trouve plus loin un article de Marco Modenesi sur «la Production du texte dans 'Fusée': G. Hénault ou le surréalisme reconquis». Qui s'étonnera de la présence sensible de $\mathrm{G}$. Hénault trouvera réponse à ses questions en lisant le compte rendu de Carla Fratta sur Segnali per $i$ veggenti, traduction de Signaux pour voyants (l'Hexagone 1972) par Liana Nissim, professeur à l'Università del Sacro Cuore de Milan. Après l'Homme rapaillé (l'Uomo rappezzato) la collection de Sergio Zoppi ( $\mathrm{Dal}$ mondo intero», Bulzoni Editore) compte donc un quatrième poète québécois (avec Giguère et $\mathrm{P}$.-M. Lapointe) en la bonne compagnie d'Apollinaire et de Tardieu.

J.A.

Tania Franco Carvalhal, LITERATURA COMPARADA,

São Paulo, éditora Atica, 1986, 88 p., serie principios 58

Tania Franco Carvalhal vient de publier un livre sur la littérature comparée dont on pourrait dire, à première vue, qu'il est petit, par le nombre de pages et la collection où il figure. La "serie principios" est l'équivalent brésilien du "Que sais-je" et constitue une sorte d'encyclopédie de poche publiée à l'intention d'un public d'étudiants et où chaque titre fait le tour d'une question de manière succinte.

Pourtant le mérite de ce livre n'est pas mince. D'abord parce qu'écrire de manière sérieuse sur un sujet de réflexion dans nos pays périphériques impose toujours un double effort. Celui de faire l'inventaire de la réflexion des autres, ceux du centre, et l'effort de dresser le bilan de notre propre contribution, à nous.

Or c'est ce que l'auteur fait, de manière succinte, mais avec brio, précision et compétence. Non seulement fait-elle l'historique du développement des études comparatistes mais elle souligne le caractère et l'orientation des premières étapes de cette science. Elle explique le contexte et les raisons de la crise récente du comparatisme. Et finalement elle fait ressortir les orientations divergentes qu'ont connues les études de littérature comparée avec les écoles américaines et esteuropéennes dans la mouvance des théories structuralistes et sémiotiques.

A chacune des étapes de cette histoire du comparatisme, elle fait surtout le point sur les apports des critiques brésiliens. On sait quel développement a connu, au Brésil, l'étude de la Théorie littéraire, à l'époque de la dictature militaire, c'est-à-dire dans les années $60-70$. En resituant ainsi à chaque fois la problématique 
abordée dans le contexte du lecteur brésilien auquel ce livre est d'abord destiné, l'auteur nous montre qu'elle réfléchit sur son objet à partir du lieu d'où elle parle. Et le dernier chapitre du livre constitue d'une certaine façon une comparaison des comparatismes, ou si l'on préfère, un regard périphérique sur les comparatismes du centre. Intitulé: «Literatura comparada et dependencia cultural», ce chapitre final se divise en des sections dont les titres éclairent fort bien le projet de l'auteur: «1- analogie et dépendance 2- différence et dépendance 3- la voracité anthropophagique 4- le comparatisme et la décolonisation littéraire».

Mettant en évidence l'eurocentrisme qui a marqué traditionnellement les études de littérature comparée, Tania Franco Carvalhal souligne la nécessité d'un nouveau sinon d'un autre comparatisme. Elle montre en tout cas, pour le Brésil, dans quelle mesure un critique comme Antonio Candido ou une théorie esthétique comme celle de «l'anthropophagie culturelle» d'Oswald de Andrade ont ouvert la voie et dégagé les perspectives d'un regard différent sur les relations littéraires du Brésil avec les autres littératures. Ces pionniers qui plus est ont fait école. Silviano Santiago, auteur de Uma literatura nos tropicos (Une littérature sous les tropiques), où l'on peut trouver des études aux titres aussi suggestifs que: «EÇA de Queiroz, auteur de Madame Bovary, «Universel malgré la dépendance», Haroldo de Campos, poète, critique et traducteur de réputation internationale, José Guilherme Melquior... sont, entre autres, comme dit l'expression à la mode, des noms avec lesquels il faut désormais compter.

Par cet enracinement de la réflexion dans un lieu bien identifié le livre de Tania Franco Carvalhal ne vaut pas seulement pour le lecteur brésilien mais nous rejoint tous. Il vaut pour nous tous qui nous préoccupons, à un titre ou à un autre, du renouvellement en profondeur, du comparatisme.

Son propos fait tout naturellement écho à ce qui se dit ailleurs, partout où de nouvelles voix se font entendre. Jean Dérive, par exemple, faisait remarquer dans un texte sur «littérature comparée: problèmes de méthodes et positions idéologiques» paru dans Littérature et méthodologie, (Abidjan, éditions CEDA, 1984) que pour l'écrivain africain utilisant une langue européenne pour traduire une culture apparentée à d'autres cultures africaines s'exprimant en des langues véhiculaires différentes et surtout en des langues européennes différentes, le problème de la comparaison pouvait présenter des complexités inconnues de la tradition européenne.

Que dans son livre Tania Carvalhal soit attentive à toutes ces voix nouvelles qui de plus en plus transforment le panorama du comparatisme traditionnel, on peut s'en convaincre à parcourir la bibliographie commentée par laquelle son livre se termine. Sur treize titres cités et commentés, cinq proviennent de la Francophonie, c'est-à-dire deux de la France hexagonale et trois de cette périphérie de la langue française que constituent: l'Afrique, la Caraïbe et le Québec. 
L'institution littéraire au Canada: perspectives historiques

Institut de Recherches en Littérature Comparée, Université de l'Alberta, Edmonton, Alberta, T6G 2E6

tél.: $403-432-2459,3647$ ou 4926

Il s'est constitué à l'Université de l'Alberta, sous l'égide de l'Institut de Recherches en Littérature Comparée, un groupe de chercheurs qui se sont proposé la tâche - ambitieuse mais éminemment réalisable - de préparer une histoire, non des littératures canadiennes, mais de l'institution littéraire telle qu'elle s'est développée au Canada depuis les origines jusqu'à nos jours. À cette fin, la division canadienne de l'Institut projette d'organiser, entre 1986 et 1990 , un certain nombre de colloques dont chacun portera sur un aspect particulier de l'institution littéraire considérée comme phénomène socio-historique. On espère établir ainsi les bases d'une théorie et d'une histoire du cadre institutionnel qui régit les conditions de production et de réception de la littérature au Canada.

Les colloques prévus par l'Institut porteront sur les sujets suivants:

1. Problèmes de réception littéraire; 2. Questions d'édition et de diffusion; 3. Préfaces et manifestes littéraires; 4. Les genres littéraires; 5 . La traduction littéraire; 6 . La littérature au féminin; 7. Traditions orales indigènes; 8 . La littérature en langues de moindre diffusion; 9 . La paralittérature; 10 . La canonisation.

Outre les actes des différents colloques, l'Institut compte publier une série d'études socio-historiques approfondies portant sur des aspects particuliers de l'institution littéraire et dont le but global sera de sonder les conditions de production et de réception de la littérature au Canada. Les collaborateurs éventuels sont priés de s'adresser aux responsables suivants (à l'adresse ci-dessus mentionnée):

Littérature canadienne comparée : E.D. Blodgett, Département de littérature comparée.

Littératures indigènes et littérature en langues de moindre diffusion: M.V. Dimic, Département de littérature comparée.

Littérature canadienne-anglaise: S.C. Neuman, Département d'anglais. Littérature canadienne-française: A.G. Purdy, Département de langues romanes. 